\title{
Validade de construto e consistência interna da escala de autoestima de Rosenberg para uma população de idosos brasileiros praticantes de atividades físicas
}

\section{Construct validity and reliability in Rosenberg's self-steem scale for Brazilian older adults who practice physical activities}

\author{
S.T. Meurer, C.B. Luft, T.R. Benedetti, G.Z. Mazo
}

ARTIGO ORIGINAL | ORIGINAL ARTICLE

\begin{abstract}
RESUMO
Objetivou-se investigar a validade de construto e a consistência interna da Escala de Autoestima de Rosenberg (RSES) (1965) para idosos brasileiros. Participaram 292 idosos (67.54; DP = 6.90 anos) praticantes de atividades físicas na cidade de Florianópolis, Santa Catarina, Brasil. Para analisar a validade de construto, realizou-se a análise fatorial, descrita em duas etapas: a análise dos componentes principais e a análise dos pesos fatoriais. Após, procedeu-se à rotação oblíqua, por meio do método Oblimin. Para verificar a consistência interna da escala, utilizou-se o coeficiente alfa de Cronbach. Observou-se a existência de dois fatores, que, juntos, explicaram $51 \%$ da variância total dos resultados da escala. O primeiro desses fatores sozinho explicou 38\% dessa variância e o segundo por apenas $13.4 \%$ da mesma. A análise fatorial sem rotação indicou que a escala pode ser considerada unidimensional. No entanto, após a rotação, verificou-se que os itens negativos constituem o primeiro fator e os positivos o segundo fator. O alfa de Cronbach verificado foi de 0.812 , considerado bom. Observou-se que a RSES apresentou qualidades psicométricas aceitáveis para a pesquisa com idosos brasileiros praticantes de atividades físicas, desta forma, pode ser considerado um instrumento válido e confiável para ser utilizado com essa população.
\end{abstract}

Palavras-chave: autoestima, idoso, validade dos testes

ABSTRACT

The present research aimed at investigating the construct validity and reliability of the Rosenberg's Selfesteem Scale (RSES) (1965) for Brazilian older adults. 292 elderly participants (67.54, DP = 6.90 years) who do physical activities in Florianopolis, Santa Catarina, Brazil. The analysis of the data was made in two steps: the analysis of the principal components and analysis of the factor loadings. To examine the construct validity we carried out the factor analysis described in two stages: principal component analysis and factor weights. After, we proceeded with the oblique rotation, using the method Oblimin. To check the reliability of the scale, we used the Cronbach's alpha. It was observed that there were two factors that together accounted for $51 \%$ of the total variance of the results of the scale. The first of these factors solely explained for $38 \%$ of the variance, and the second by only $13.4 \%$ of the variance. The factor analysis without rotation indicated that the scale can be considered one-dimensional. However, after the rotation, it was found that the negatives are the first factor and the second positive factor. Cronbach's alpha was established 0,812 , considered good. It was observed that the RSES had acceptable psychometric qualities for research on Brazilian elderly who practice physical activities, thus can be considered a valid and reliable instrument for use with this population.

Keywords: self-esteem, aged, validity of tests

Submetido: 07.09.2011 | Aceite: 03.02.2012

Simone Teresinha Meurer e Tânia Rosane Bertoldo Benedetti. Núcleo de Pesquisa em Cineantropometria e Desempenho Humano - Universidade Federal de Santa Catarina, Florianópolis, SC, Brasil.

Caroline Di Bernardi Luft. Laboratório de Neurociência do Esporte e Exercício \& Laboratório de Educação Cerebral - Universidade Federal de Santa Catarina, Florianópolis, SC, Brasil.

Giovana Zarpellon Mazo. Laboratório de Gerontologia - Universidade do Estado de Santa Catarina, Florianópolis, SC, Brasil.

Endereço para correspondência: Simone Teresinha Meurer, Rua Perdigão Malheiros, 294. $\mathrm{Apt}^{\mathrm{O}}:$ 202. Bairro Cidade Jardim, Belo Horizonte/MG, CEP 38380-050, Brasil.

E-mail: simonemeurer@yahoo.com.br 
O envelhecimento é acompanhado por perdas que contribuem para a redução da satisfação com o próprio corpo (Gripo \& Hill, 2008), fato que pode estar relacionado às perdas físicas, funcionais e sociais (Schieman \& Campbell, 2001).

Associado às perdas inerentes ao envelhecimento, a inatividade física age como acelerador desse processo, resultando em incapacidade funcional e perda de qualidade de vida. A inatividade física tende a aumentar com o envelhecimento (U.S. Department of Health and Human Services, 1996). Dados de pesquisa recente mostram que $53.2 \%$ das mulheres e $51.5 \%$ dos homens brasileiros acima dos 65 anos são insuficientemente ativos (Ministério Brasileiro da Saúde, 2009).

A autoestima é definida como o sentimento, o apreço e a consideração que uma pessoa sente por si própria (Rosenberg, 1965) e é destacada como uma das chaves para resolver a baixa participação em atividades físicas (Spence, McGannon, \& Poon, 2005). Partindo desse pressuposto, um estudo mostra que quanto mais alta a autoestima maior a possibilidade de inserção em programas de exercícios físicos (Wilson \& Rodgers, 2002). Também Fox (1997) relatou que a autoestima elevada está associada com a escolha, a persistência e o sucesso de comportamentos relacionados à saúde.

O modelo do exercício físico e da autoestima descrito por Sonstroem e Morgan (1989) sugere que a autoestima global e o exercício físico estejam associados por meio das percepções da autoeficácia, da competência física e da aceitação física. O exercício físico influencia na percepção da auto-eficácia, da competência e aceitação física, interferindo sobre a autoestima global (Spence et al., 2005).

A autoestima é um aspecto fundamental na vida do idoso e representa uma das dimensões da personalidade que influencia o bem-estar do indivíduo e a sua adaptação no mundo que o circunda (Ruibytè, 2007), sendo importante para o sucesso e a satisfação com a vida (Rosen- berg, 1965). É um atributo profundamente individual, embora modelado nas relações cotidianas e também decisivo na relação do indivíduo consigo mesmo e com os outros, influenciando na percepção dos acontecimentos e das pessoas, no comportamento e nas vivências do indivíduo (Andrade, Souza, \& Minayo, 2009).

Embora evidenciada a importância da autoestima para o bem-estar individual e a sua estreita relação com a atividade física, há escassez de estudos sobre a temática no cenário brasileiro, especialmente entre idosos. Um dos obstáculos para as investigações desse construto é a falta de instrumentos de medida de autoestima consolidados cientificamente.

Entre os instrumentos disponíveis, em português do Brasil, está a Escala de Autoestima de Rosenberg - RSES (Rosenberg Self-Esteem Scale) que foi desenvolvida por Morris Rosenberg (Rosenberg, 1965). Ela é uma escala para a avaliação da autoestima na população geral. Essa escala é a mais utilizada nos estudos internacionais com diferentes populações (Baranik et al., 2008), desde adolescentes (Farruggia, Chen, Greenberger, Dmitrieva, \& Macek, 2004), estudantes universitários (Pruessner et al., 2005; Tafarodi \& Milne, 2002) até idosos (An, An, O'Connor, \& Wexler, 2008; Pruessner et al., 2005). No Brasil, foram encontradas duas traduções dessa escala para o português do Brasil, uma adaptada para adolescentes (Avanci, Assis, Santos, \& Oliveira, 2007) e outra para pacientes pré-cirurgia plástica (Dini, 2000).

O estudo de Avanci, Assis, Santos, e Oliveira (2007) objetivou padronizar a escala de autoestima de Rosenberg para adolescentes residentes no Rio de Janeiro. As seguintes equivalências foram avaliadas: conceitual e de itens, semântica (significado referencial e geral), de mensuração (confiabilidade teste-reteste, consistência interna, análise fatorial e validade de construto), operacional e funcional. Foram encontrados bons resultados para equivalência semântica, o alfa de Cronbach foi de 
0.68, Kappa predominantemente moderado e regular e a análise fatorial proposta é de duas estruturas fatoriais (baixa e alta autoestima), indicando a aplicabilidade da escala na população de referência, porém sugerindo a necessidade do desenvolvimento de outros trabalhos em amostras distintas.

O objetivo do trabalho de Dini (2000) foi traduzir para a língua portuguesa, adaptar ao contexto cultural brasileiro e validar a escala de autoestima de Rosenberg para uma população de pacientes que iriam submeter-se à cirurgia plástica. Após o processo de tradução, a validade foi testada por julgamento (face e conteúdo) e por testes de correlação (Pearson, Spearman e regressão múltipla) dos escores da escala com os resultados de outras escalas (SF-36, SRQ-20, escalas numéricas de beleza, vergonha, satisfação com a aparência e cuidados com a saúde) que medem aspectos correlatos à autoestima. Observou-se um coeficiente de reprodutibilidade intra e inter-observador de 0,98. Foram identificados bons índices de reprodutibilidade e validade e o autor concluiu ser um instrumento válido para medir a autoestima de pacientes brasileiros que irão submeter-se à cirurgia plástica, com média de idade de 40 anos.

Nesse estudo, optou-se por utilizar como base a escala traduzida e adaptada por Dini (2000), pois o objetivo dessa pesquisa é validar a escala de autoestima de Rosenberg para idosos brasileiros (Tabela 1) e a média de idade dos participantes da escala adaptada por Dini (2000) foi de 40 anos, sendo mais aproximada da faixa etária dos sujeitos visados no presente estudo.

Face ao exposto, o presente estudo objetivou investigar a validade de construto e a consistência interna da Escala de Autoestima de Rosenberg (1965) para uma população de idosos brasileiros praticantes de atividade física.

\section{MÉTODO}

\section{Amostra}

Participaram do estudo idosos integrados em programas de atividades físicas na Universidade Federal de Santa Catarina e na Prefeitura Municipal de Florianópolis-SC. Os programas de atividades físicas eram desenvolvidos com aulas de ginástica, realizadas três vezes por semana, com duração de uma (1) hora por sessão, com orientação de profissionais formados em Educação Física. Nessas aulas eram trabalhadas diferentes qualidades físicas, entre elas a flexibilidade, a força, o equilíbrio, a coordenação e a resistência aeróbia, além de exercícios específicos para o fortalecimento da musculatura pélvica e a descontração por meio de relaxamento.

Os critérios de inclusão foram: apresentar mais de 60 anos e ter disponibilidade em participar do estudo. Assim, participaram do estudo

Tabela 1.

Escala de autoestima de Rosenberg para idosos brasileiros praticantes de atividades físicas

\begin{tabular}{|c|c|c|c|c|}
\hline & $\begin{array}{l}\text { Concordo } \\
\text { plenamente }\end{array}$ & Concordo & Discordo & $\begin{array}{l}\text { Discordo } \\
\text { plenamente }\end{array}$ \\
\hline Em geral, estou satisfeito comigo mesmo (a). & 4 & 3 & 2 & 1 \\
\hline Às vezes, eu acho que eu não sirvo para nada. & 1 & 2 & 3 & 4 \\
\hline Eu sinto que eu tenho um tanto de boas qualidades. & 4 & 3 & 2 & 1 \\
\hline $\begin{array}{c}\text { Eu sou capaz de fazer coisas tão bem quanto a maioria das } \\
\text { outras pessoas. }\end{array}$ & 4 & 3 & 2 & 1 \\
\hline Eu sinto que não tenho muito do que me orgulhar. & 1 & 2 & 3 & 4 \\
\hline Às vezes, eu realmente me sinto inútil. & 1 & 2 & 3 & 4 \\
\hline Eu sinto que sou uma pessoa de valor, igual às outras pessoas. & 4 & 3 & 2 & 1 \\
\hline Eu gostaria de ter mais respeito por mim mesmo (a). & 1 & 2 & 3 & 4 \\
\hline $\begin{array}{c}\text { Quase sempre eu estou inclinado (a) a achar que sou um(a) } \\
\text { fracassado (a). }\end{array}$ & 1 & 2 & 3 & 4 \\
\hline Eu tenho uma atitude positiva em relação a mim mesmo (a). & 4 & 3 & 2 & 1 \\
\hline
\end{tabular}


292 idosos (235 mulheres e 57 homens), com média de idade de 67.54 anos $(\mathrm{DP}=6.90)$, participantes de programas de atividades físicas na Universidade Federal de Santa Catarina $(n=$ $188)$ e na Prefeitura Municipal de Florianópolis $(n=104)$.

\section{Instrumentos}

A Escala de Autoestima de Rosenberg RSES foi desenvolvida por Rosenberg em 1965 é um instrumento utilizado para a avaliação da autoestima global (Rosenberg, 1965). Para o autor, a autoestima é a autoavaliação pessoal, a qual implica um sentimento de valor, que engloba um componente predominantemente afetivo, expresso numa atitude de aprovação/ desaprovação em relação a si mesma.

A RSES é constituída por 10 itens, com conteúdos referentes aos sentimentos de respeito e aceitação de si mesmo. Metade dos itens é enunciada positivamente e a outra metade negativamente. Para cada afirmação existem quatro opções de resposta, tipo Likert (concordo totalmente $=4$, concordo $=3$, discordo $=2$ e discordo totalmente $=1$ ). A soma das respostas aos 10 itens fornece o escore da escala cuja pontuação total oscila entre 10 e 40 e a obtenção de uma pontuação alta reflete uma autoestima elevada.

Foi realizado um estudo piloto com 10 idosos com características semelhantes da amostra, utilizando a RSES (Rosenberg, 1965) traduzida e adaptada por Dini (2000). Nessa aplicação, os idosos foram entrevistados e foi observada a compreensão da escala. Além da resposta ao item especificamente, foi aplicada uma escala de clareza, questionando se a questão estava "clara", "mais ou menos clara" ou "nada clara".

Uma das questões propostas no questionário sofreu ajuste, uma vez que a maioria dos idosos teve dificuldade de entendê-la, passando de "Eu sinto que sou uma pessoa de valor, pelo menos num plano igual às outras pessoas" para "Eu sinto que sou uma pessoa de valor, igual às outras pessoas". A questão proposta também foi avaliada em relação à sua clareza, pelos idosos, sendo que foi considerada "clara".

\section{Procedimentos}

Este estudo foi aprovado pelo Comitê de Ética na Pesquisa em Seres Humanos da Universidade Federal de Santa Catarina $\left(n^{\circ}\right.$. 075/2008) e pela Universidade do Estado de Santa Catarina (UDESC) ( ${ }^{\circ}$. 185/2007). Todos os idosos participantes dos programas de atividade física mencionados foram convidados a participar do estudo e assinaram o Termo de Consentimento Livre e Esclarecido (TCLE).

A coleta de dados ocorreu em forma de entrevista individual, por entrevistadores previamente treinados, nas dependências físicas do ginásio de esportes da UDESC e nos Centros de Saúde do município de Florianópolis, locais onde ocorriam as atividades físicas.

\section{Análise Estatística}

Após a tabulação e organização dos dados, o banco de dados foi conferido visando identificar possíveis erros de digitação. Analisou-se a normalidade dos dados a partir de histogramas e gráficos de caixa, visando identificar as características do escore geral (assimetria e curtose), bem como testar a sua normalidade (Kolmogorov-Smirnov).

A análise estatística descritiva dos dados (medidas de tendência central e variabilidade) foi feita com o escore geral, com o grupo todo e separado por sexo. Isso foi feito para explorar a capacidade da escala em diferenciar os grupos, bem como entender como é a distribuição dos dados para o sexo feminino e masculino.

Para analisar a validade de construto, realizou-se a análise fatorial exploratória dos dados. A análise fatorial exploratória permite investigar os padrões de correlação entre as questões da escala, visando identificar o construto latente às questões, especialmente em relação à sua dimensionalidade. Primeiramente, procedeu-se a análise do índice de Keiser-Meyer 
Olkin (KMO) para verificar a adequação dos dados para análise fatorial. Essa é uma medida da fatorabilidade das matrizes de correlação na qual a análise fatorial está baseada. Em seguida, realizou-se o teste de esfericidade de Bartlett para verificar se os dados atendiam ao pré-requisito de esfericidade.

A análise fatorial foi descrita em duas etapas: a análise dos componentes principais e a análise dos pesos fatoriais. A análise dos componentes principais foi feita para identificar quantos fatores ou dimensões são responsáveis pela variação nos itens da escala e o quanto de variância cada um desses fatores consegue explicar (em percentual com base nos valores próprios - eigenvalues). Nessa fase, o objetivo foi obter um número mínimo de fatores que expliquem o máximo da variação no conjunto total dos dados. Além disso, nessa fase se observa os valores das comunalidades, que indicam o quanto de variância foi extraída, ou seja, quanto de variância essa variável compartilha com o conjunto total.

Para determinar o número de fatores a serem retidos, utilizou-se dois critérios combinados: o dos autovalores (>1) e o scree plot. Ou seja, buscou-se o equilíbrio entre os eigenvalues e o ponto onde eles estabilizam, chamado como cotovelo do gráfico. Após, procedeu-se à rotação oblíqua, por meio do método Oblimin (delta em zero). Optou-se por um método de rotação oblíqua, porque a escala mede apenas um construto ou então dimensões que apresentam correlação entre si. Observou-se a pattern matrix porque ela costuma prover uma interpretação melhor dos fatores e cargas. Conforme geralmente praticado na literatura
(Streiner \& Norman, 2007), itens que apresentaram pesos fatoriais acima de 0.3 foram considerados, sendo que aquelas acima de 0.4 foram consideradas significativas. Para verificar a consistência interna da escala, utilizou-se o coeficiente alfa de Cronbach.

\section{RESULTADOS}

Em relação ao escore total da escala, observou-se uma assimetria negativa (assimetria $=-0.922$ ) e uma curtose positiva menos acentuada (curtose $=0.491$ ). Isso significa que as pessoas tendem a apresentar escores máximos na escala, o que indica a presença de um efeito "teto" moderado. Por essa razão, o teste de normalidade de Kolmogorov-Smirnov apontou que a distribuição difere significativamente de uma distribuição normal $(p<.001)$.

As medidas de tendência central e variabilidade são apresentadas na Tabela 2.

Em relação à análise fatorial (Tabela 3) exploratória, foram atingidos os pré-requisitos para a sua realização $(\mathrm{KMO}=0.848$; Barlett $=$ 759.98, $p<.001$ ). Esses indicadores apontam que os dados são apropriados para a análise fatorial.

Primeiramente, na análise dos componentes principais, observou-se a existência de dois fatores (com autovalores $>1$ ), que juntos responderam por $51 \%$ da variância total dos resultados da escala. O primeiro desses fatores, sozinho, respondeu por $38 \%$ dessa variância e o segundo por apenas $13.4 \%$ da mesma. Isso indica que a escala mede predominantemente o primeiro fator. Como era presumido que a escala é unidimensional, optou-se pela solução fatorial com um único fator, pois todos os itens

Tabela 2.

Medidas de tendência central, variabilidade e distribuição do escore total na escala Rosenberg.

\begin{tabular}{cccccccc}
\hline Escore & $\mathrm{N}$ & \multirow{2}{*}{ Média (DP) } & Min-Max & I.C. (95\%) & Assim. & Curt. & $p^{*}$ \\
\hline Todos & 292 & $35.10(4.18)$ & $19-40$ & $34.6-35.6$ & -0.922 & 0.491 & $<.001$ \\
\hline Masculino & 57 & $35.09(4.12)$ & $26-40$ & $34.0-36.14$ & -0.564 & -0.658 & $<.001$ \\
Feminino & 235 & $35.11(4.24)$ & $22-40$ & $34.5-35.65$ & -0.993 & 0.697 & $<.001$ \\
\hline
\end{tabular}

* Esse valor de $p$ é resultado do teste de normalidade Kolmogorov-Smirnov, valores abaixo de .05 indicam que a distribuição difere significativamente de uma distribuição normal. 
Tabela 3.

Pesos fatoriais e comunalidades dos itens da escala com e sem rotação oblíqua.

\begin{tabular}{|c|c|c|c|c|}
\hline \multirow[b]{2}{*}{ Item } & Solução 1 & \multicolumn{2}{|c|}{ Solução 2} & \multirow[b]{2}{*}{$h^{2}$} \\
\hline & Autoestima & P. Negativa & P. Positiva & \\
\hline $\begin{array}{l}\text { Quase sempre eu estou inclinado (a) a achar que sou um } \\
\text { fracassado }\end{array}$ & 0.722 & 0.697 & - & 0.566 \\
\hline Às vezes, eu realmente me sinto inútil & 0.696 & 0.878 & - & 0.694 \\
\hline Eu tenho uma atitude positiva em relação a mim mesmo (a). & 0.651 & - & 0.603 & 0.521 \\
\hline Às vezes, eu acho que eu não sirvo para nada. & 0.644 & 0.815 & - & 0.596 \\
\hline Eu gostaria de ter mais respeito por mim mesmo (a). & 0.644 & 0.641 & - & 0.459 \\
\hline Eu sinto que não tenho muito do que me orgulhar. & 0.638 & 0.589 & - & 0.430 \\
\hline Em geral, estou satisfeito comigo mesmo (a). & 0.631 & 0.395 & 0.366 & 0.405 \\
\hline Eu sinto que eu tenho um tanto de boas qualidades. & 0.540 & & 0.829 & 0.639 \\
\hline \multirow{2}{*}{$\begin{array}{c}\text { Eu sinto que sou uma pessoa de valor, igual às outras pessoas. } \\
\text { Eu sou capaz de fazer coisas tão bem quanto a maioria das } \\
\text { outras pessoas }\end{array}$} & 0.517 & - & 0.577 & 0.387 \\
\hline & 0.440 & - & 0.710 & 0.459 \\
\hline Alfa de Cronbach & 0.812 & 0.786 & 0.686 & \\
\hline Percentual de Variância Explicada & $38 \%$ & $38 \%$ & $13.4 \%$ & \\
\hline
\end{tabular}

carregam significativamente no primeiro fator (38\%). Apresentou-se nessa mesma Tabela as cargas dos itens nos dois fatores, utilizando rotação oblimin.

Embora se tenha verificado a existência de dois fatores, observou-se que, sem rodar a matriz, todos os itens carregam significativamente em um único fator, todos com cargas acima de 0.4. Ao rodar a matriz, são separados os itens positivos e negativos da escala, mostrando que existe, na escala, duas dimensões, uma relacionada com percepção positiva de si mesmo, e outro negativa. O mais forte é o negativo, o que indica uma tendência da escala a valorizar os aspectos negativos da autoestima.

Em relação à consistência interna, o alfa de Cronbach verificado foi de 0.812 , o que se pode considerar aceitável para uma escala.

\section{DISCUSSÃO E CONCLUSÕES}

A maioria dos estudos que avaliaram as propriedades psicométricas da RSES (1965) utilizaram análise fatorial exploratória (Andrade et al., 2009; Avanci et al. 2007; Baranik et al., 2008; Dias, 1996; Schmitt \& Allik 2005) ou confirmatória (Farruggia et al., 2004; Horan, DiStefano, \& Motl, 2003; Romano, Negreiros, \& Martins, 2007).

A pontuação média obtida com os participantes do presente estudo foi elevada (35.10;
$\mathrm{DP}=4.28)$, corroborando com Schmitt e Allik (2005) que, ao investigarem a autoestima de pessoas de 53 diferentes nações, utilizando a RSES, também identificaram uma média elevada (30.85; DP $=4.82$ ). De acordo com Schmitt e Allik (2005), as pontuações foram acima do ponto médio teórico do RSES, desta forma sugerindo que a autoestima geralmente positiva pode ser universal. Acredita-se que a autoestima elevada também possa ser atribuída à participação em atividades físicas, conforme já vem sendo apontado em outros estudos (Chodzko-Zajko et al., 2009; Spence et al., 2005). No entanto, a análise da distribuição dessa escala indicou que a autoestima talvez esteja sendo medida com questões que induzem respostas de escores elevados, pois verificou-se um efeito teto no instrumento, conforme foi demonstrado nos resultados. $\mathrm{O}$ efeito teto indica que a maioria das pessoas apresenta escores perto do máximo, o que sugere a presença de algum viés na escala. Considera-se que o enviesamento possa estar relacionado com a maneira como as pessoas respondem à escala. Adicionar mais graduações nas respostas, tais como 0 a 10, poderia auxiliar na variabilidade das mesmas.

A estrutura fatorial da RSES vem sendo estudada e alguns estudos consideram a escala unidimensional (Azevedo \& Faria, 2004; 
Schmitt \& Allik, 2005), conforme também mostra o presente estudo, enquanto que outros a consideram bidimensional (Andrade et al., 2009; Dias, 1996; Romano et al., 2007).

Os resultados do presente estudo corroboram com Rosenberg (1979) que evidenciou que estes fatores (positivos e negativos) medem o mesmo construto, baseando-se no fato de que os padrões de correlação com diversas variáveis são praticamente similares (Tafarodi \& Milne, 2002). Esse argumento de Rosenberg (1979) ajuda a explicar porquê todos os itens carregam significativamente em um único fator (cargas acima de 0.4) e também puderam ser separados a partir da rotação da matriz. Desta forma, evidencia-se que a estrutura mais adequada seja unidimensional, uma vez que a escala se propõe a medir a autoestima global e sugere a soma de valores e não a distinção entre autoestima positiva e negativa (Greenberger, Chen, Dmitrieva, \& Farruggia, 2003).

Marsh (1996) observou que a RSES (1965) pode ser unidimensional numas populações e bidimensional noutras, pois de acordo com Goldsmith (1986) e Marsh (1996), as variações observadas na estrutura fatorial podem estar relacionadas com as características das amostras utilizadas, como as variáveis sexo e idade dos sujeitos, capacidade de compreensão e tipo de ocupação.

Dias (1996), em uma validação com jovens portugueses, encontrou dois fatores com autovalores superiores a 1 (4.53 e 1.23), que explicavam $57.7 \%$ da variância total da RSES. De forma idêntica, no presente estudo também foram verificadas duas dimensões (com autovalores $>1$ ), sendo uma relacionada com percepções positivas e outra com percepções negativas, explicando $51 \%$ da variância total dos resultados.

Um estudo realizado com adultos brasileiros (Andrade et al., 2009) concluiu que a RSES (1965) explicou 59\% da variância total dos resultados, sendo que a escala apresentou dois fatores. De forma semelhante ao presente estudo, no primeiro fator, concentraram-se itens da avaliação predominantemente negativa e, no segundo, de avaliação positiva, indicando uma tendência da escala a valorizar os aspectos negativos da autoestima.

Tafarodi e Milne (2002), aplicando a RSES a estudantes do curso de psicologia, explicaram $61 \%$ da variância total ao fator comum e $22 \%$ aos fatores positivos e negativos. No presente estudo com idosos brasileiros, o total da variância explicada foi inferior (51\%), sendo que o primeiro fator respondeu por $38 \%$, indicando que a escala mede predominantemente o primeiro fator, ou seja, quando tratada de forma unidimensional.

Em uma pesquisa mais abrangente (Schmitt \& Allik, 2005) realizada com pessoas de 53 nações, a proporção da variação explicada para o primeiro componente principal variou de 24.6\%, na República Democrática do Congo, a $54.4 \%$, em Israel, com $41.4 \%$ da variação explicada quando consideradas todas as 53 nações. Comparando os resultados, o percentual da variância explicada no presente estudo foi superior (51\%).

Em estudo realizado com adolescentes portugueses (Romano et al., 2007), o total de variância explicada foi de 46,03\%. Assim, pode-se considerar que a variância explicada pela RSES no presente estudo realizado com idosos brasileiros $(51 \%)$ pode ser considerada satisfatória.

Ao encontro de outros estudos (Andrade et al., 2009; Marsh, 1996; Santos \& Maia, 1999, 2003), verificou-se tendência de valorização das questões negativas que sugerem que as respostas aos itens negativos, mesmo após a sua inversão, produzem fatores independentes, o que pode significar que os sujeitos não interpretam da mesma maneira os itens formulados negativamente. Assim, concorda-se com Corwyn (2000) e Dunbar, Ford, Hunt, e Der (2000) que apontam que estes dois fatores são, na maioria das vezes, uma conseqüência dos efeitos do método associados com os itens 
negativamente expressos.

Esses resultados vão ao encontro dos encontrados na presente pesquisa, uma vez que os itens com os maiores pesos fatoriais são em sua maioria negativos (ex. Quase sempre eu estou inclinado (a) a achar que sou um fracassado; às vezes, eu realmente me sinto inútil; às vezes, eu acho que eu não sirvo para nada; eu gostaria de ter mais respeito por mim mesmo (a); eu sinto que não tenho muito do que me orgulhar).

A escala contém itens negativos e positivos porque um dos métodos para construir escalas indica que é importante incluir afirmações em ambos os sentidos para reduzir a tendência de respostas positivas em todos os itens (DeVe1lis, 2003). Esse método pressupõe que tanto os itens positivos quanto os negativos representam o mesmo construto, no entanto, a confiabilidade dessas escalas normalmente é reduzida devido a estrutura bifatorial que elas geram (Quilty, Oakman, \& Risko, 2006).

O estudo realizado por Quilty et al. (2006) indicou que fatores de personalidade estão diferentemente associados com as respostas aos itens negativos e positivos. Por exemplo, quanto maior o neuroticismo do sujeito, maior a sua tendência a responder mais nas afirmações negativas e menos nas positivas. Como existem diversos padrões de personalidade, os autores sugerem que essas escalas devem sim manter a estrutura de itens positivos e negativos, justamente para evitar que a personalidade afete muito no escore total ou mascare as diferenças entre grupos.

Por exemplo, em estudos com atividade física, se a escala só contiver itens negativos, ao comparar o grupo sedentário com o grupo ativo, e a autoestima do grupo ativo fosse significativamente mais alta, não se saberia ao certo o quanto o neuroticismo ou as diferenças de personalidade entre os grupos afetaram o resultado. Dessa forma, conforme apontam Quilty et al. (2006) sugere-se a utilização da escala com o somatório das questões positivas e negativas, mas também se considera válida a exploração dos dois fatores embora o somatório total seja mais válido, conforme foi argumentado e investigado pelos autores.

Analisando os itens separadamente, identifica-se na literatura (Baranik et al., 2008; Farruggia et al., 2004) que o item "eu gostaria de ter mais respeito por mim mesmo (a)" é o que normalmente tem baixa carga fatorial, contrapondo os achados do presente estudo. Ainda, esse mesmo item pode ter carregamento positivo ou negativo, dependendo do contexto de análise, sendo considerado ambíguo. De forma idêntica aos resultados do presente estudo, na análise das versões da RSES (1965) para diferentes países (Bangladesh, Republica Democrática do Congo, Indonésia, Malásia e Tanzânia), também tiveram carregamentos negativos para este item (Schmitt \& Allik, 2005).

O item "em geral, estou satisfeito comigo mesmo(a)", carregou tanto no fator positivo quanto negativo (cross loading), o que ainda não foi documentado em outros estudos que analisaram a RSES. Todavia, esse mesmo item apresentou baixos pesos fatoriais para amostras de diferentes países (Canadá, Alemanha, Nova Zelândia, Kenya, Singapura e Taiwan), conforme registrado por Baranik et al. (2008). No estudo realizado com adolescentes portugueses (Romano et al., 2007), esse item teve baixo carregamento (0.41) e se enquadrou naqueles de avaliação positiva. Dessa forma, sugere-se que a compreensão de estar satisfeito remeteu a um significado negativo para muitos dos idosos participantes do presente estudo. Pode-se sugerir que tiveram a interpretação que "estar satisfeito" representaria não buscar melhorar em algum aspecto, ou seja, esse item pode ter sido interpretado de forma ambígua pelos idosos.

No estudo de Schmitt e Allik (2005), o item "Eu sinto que sou uma pessoa de valor, igual às outras pessoas" teve carga fatorial com variação de 0.53 à 0.80 . O resultado identificado para este item no presente estudo é aproximado e pode ser considerado aceitável. 
Todavia observa-se que a comunalidade desse item é baixa. Essa questão sofreu modificação no processo de adaptação para idosos e sugere-se que ainda não esteja suficientemente clara, uma vez que pode ter interpretação dúbia, pois o sujeito pode se considerar com "valor superior", o que o faria discordar completamente da questão e pontuar o valor mínimo, mesmo tendo um apreço elevado por si próprio.

O item "Eu sou capaz de fazer coisas tão bem quanto a maioria das outras pessoas" apresentou menor carga fatorial na presente pesquisa (0.440). O mesmo item teve carregamento semelhante para alguns países no estudo conduzido por Schmitt e Allik (2005), sendo que a carga fatorial variou de 0.37 (França) a 0.79 (Bangladesh). No Brasil, o estudo citado identificou carga fatorial de 0.48 neste item, resultado próximo à presente investigação. Sugere-se que esta questão também pode ter duplicidade de interpretação, uma vez que a resposta do idoso depende do parâmetro de comparação que utilizou. Se considerar como "outras pessoas", aqueles da mesma faixa etária, a resposta poderia ser positiva, mas, se considerar pessoas mais jovens, por exemplo, sua resposta poderia ser negativa.

A confiabilidade da escala foi avaliada pela consistência interna, verificada por meio do coeficiente Cronbach alfa. Em um estudo que avaliou a versão traduzida da RSES para 53 países (Schmitt \& Allik, 2005), foi identificado que a confiabilidade média transversal de todas as nações foi 0.81 , o que se assemelha ao valor do alfa obtido na presente investigação (0.812).

No pesquisa de Schmitt e Allik (2005), a mais baixa consistência interna foi encontrada na República Democrática do Congo (0.45) e o mais elevado foi compartilhado por Israel e Reino Unido (0.90). Para a versão brasileira avaliada no mesmo estudo, o valor de alfa de Cronbach foi de 0.79 , ligeiramente inferior ao identificado com a presente pesquisa realizada com idosos.

Assis, Pesce, e Avanci (2006) encontraram alfa de Cronbach de 0.81 para a Escala de Rosenberg aplicada em adolescentes. Giacomoni (2002), investigando o bem-estar subjetivo de crianças, encontrou índice inferior à presente pesquisa (0.75 de Alpha de Cronbach). Gonzalez, Fortaleza, e Ruiz (1993), adaptando a escala de Rosenberg para adolescentes mexicanos, obtiveram alfa de Cronbach de 0.67. No estudo da RSES com adolescentes portugueses (Romano et al., 2007), o alfa identificado foi de 0.63. Assim, o coeficiente alfa de Cronbach obtido na presente pesquisa pode ser considerado aceitável para essa escala, pois os seus valores são próximos ou superiores aos encontrados nas versões citadas na literatura.

Considerando que a solução com um único fator mostrou-se adequada, tanto em termos de cargas fatoriais quanto em termos de consistência interna, recomenda-se o uso da estrutura de um único fator. Além disso, os valores de consistência interna das duas dimensões encontradas na segunda solução são inferiores ao que foi encontrado para a primeira solução. Dessa forma, recomenda-se que o uso da escala com idosos ativos seja feito com base no construto unitário da escala, ou seja, com base na solução 1. Sugere-se a necessidade da revisão de algumas questões e um novo estudo das qualidades psicométricas da escala.

\section{Agradecimentos:}

Nada declarado.

\section{Conflito de Interesses:}

Nada declarado.

\section{Financiamento:}

Nada declarado.

\section{REFERÊNCIAS}

An, J., An, K., O'Connor, L., \& Wexler, S. (2008). Life satisfaction, self-esteem, and perceived health status among elder Korean women: 
Focus on living arrangements. Journal of Transcultural Nursing, 19, 151- 160.

Andrade, E. R., Sousa, E. R., \& Minayo, M. C. S. (2009). Intervenção visando a autoestima e qualidade de vida dos policiais civis do Rio de Janeiro. Ciência \& Saúde Coletiva, 14, 275-285.

Assis, S. G., Pesce, R. P., \& Avanci, J. Q. (2006). Resiliência: Enfatizando a Proteção na Adolescência. Porto Alegre, RS: Artmed.

Avanci, J. Q., Assis, S. G., Santos, N. C., \& Oliveira, R. V. C. (2007) Adaptação transcultural de escala de autoestima para adolescentes. Psicologia: Reflexão e Crítica, 20, 397-405.

Azevedo, A. S., \& Faria, L. A. (2004). Autoestima no ensino secundário: Validação da Rosenberg Self-Esteem Scale. In C. Machado, L. S. Almeida, M. Gonçalves, \& V. Ramalho (Eds.), Actas da X Conferência Internacional de Avaliação Psicológica: Formas e Contextos (pp.415-421). Braga: Psiquilíbrios.

Baranik, L. E., Meade, A. W., Lakey, C. E., Lance, C. E., Hu, C., Hua, W., \& Michalos, A. (2008). Examining the differential item functioning of the Rosenberg self-esteem scale across eight countries. Journal of Applied Social Psychology, 38, 1867-1904.

Chodzko-Zajko, W. J., Proctor, D. N., Singh, M. A. F., Minson, C. T., Nigg, C. R., Salem, G. J, \& Skinner, J. S. (2009). Exercise and physical activity for older adults. Medicine and Science in Sports and Exercise, 41, 1510-1530.

Corwyn, R. F. (2000). The factor structure of global self-esteem among adolescents and adults. Journal of Research in Personality, 34, 357-379.

DeVellis, R. F. (2003). Scale Development: Theory and Applications (2nd ed.). London: SAGE Publications.

Dias, M. G. F. (1996). Tarefas desenvolvimentais e bem-estar psicológico dos jovens (Tese de Doutoramento não-publicada). Universidade do Porto, Porto, Portugal.

Dini, G. M. (2000). Tradução para a língua portuguesa, adaptação cultural e validação do questionário de autoestima de Rosenberg (Dissertação de Mestrado). Universidade Federal de São Paulo,
São Paulo, Brasil.

Dunbar, M., Ford, G., Hunt, K., \& Der, G. (2000). Question wording effects in the assessment of global self-esteem. European Journal of Psychological Assessment, 16, 13-19.

Farruggia, S. P., Chen, C., Greenberger, E., Dmitrieva, J., \& Macek, P. (2004). Adolescent self-esteem in cross-cultural perspective: Testing measurement equivalence and a mediation model. Journal of Cross-Cultural Psychology, 35, 719-733.

Fox, K. R. (1997). The physical self and processes in self-esteem development. In Kenneth R. Fox (Editor), The Physical Self: From Motivation to Well-Being (pp.111-139). Champaign, IL: Human Kinetics.

Giacomoni, C. H. (2002). Bem-estar subjetivo infantil: Conceito de felicidade e construção de instrumentos para avaliação (Tese de Doutoramento não-publicada). Universidade Federal do Rio Grande do Sul, Porto Alegre, Brasil.

Goldsmith, R. E. (1986). Dimensionality of Rosenberg self-esteem scale. Journal of Social Behavior and Personality, 1, 253-264.

Gonzalez-Fortaleza, C. F., \& Ruiz, E.R. (1993). Autoestima y sintomatología depresiva en adolescentes mexicanos: Um estudio correlacional por género. Revista ABP-APAL, 15, 59-64.

Greenberger, E., Chen, C., Dmitrieva, J., \& Farruggia, S. P. (2003). Item-wording and the dimensionality of the Rosenberg self-esteem scale: Do they matter? Personality and Individual Differences, 35, 1241-1254.

Grippo, K. P., \& Hill, M. S. (2008). Self-objectification, habitual body monitoring, and body dissatisfaction in older European American women: Exploring age and feminism as moderators. Body Image, 5, 173-182.

Horan, P. M., DiStefano, C., \& Motl, R. W. (2003). Wording effects in self-esteem scales: Methodological artifact or response style? Structural Equation Modeling, 10, 435-455.

Marsh, H. W. (1996). Positive and negative global self-esteem: A substantively meaningful distinction or artifacts? Journal of Personality and Social Psychology, 70, 810-819. 
Ministério Brasileiro da Saúde (2009). Vigitel Brasil 2008: Vigilância de fatores de risco e proteção para doenças crônicas por inquérito telefônico. Brasília, DF: Autor.

Pruessner, J. C., Baldwin, M. W., Dedovic, K., Renwick, R., Mahani, N. K., Lord, C., Meaney, M., \& Lupienb, S. (2005). Self-esteem, locus of control, hippocampal volume, and cortisol regulation in young and old adulthood. NeuroImage, $28,815-826$.

Quilty, L. C., Oakman, J. M., \& Risko, E. (2006). Correlates of the Rosenberg self-esteem scale method effects. Structural Equation Modeling, 13, 99-117.

Romano, A., Negreiros, J., \& Martins, T. (2007). Contributos para a validação da escala de autoestima de Rosenberg numa amostra de adolescentes da região interior norte do país. Psicologia, Saúde \& Doenças, 8, 109-116.

Rosenberg, M. (1965). Society and the adolescent self image. Princeton: Princeton University Press.

Rosenberg, M. (1979). Conceiving the self. New York: Basic Books.

Ruibytè, L. (2007). Relationship between individual attributional style, self-esteem, locus of control and academic achievement of vytautas magnus university students. Ugdymas, Kûno Kultûra Sportas, 4, 71-78.

Santos, P. J., \& Maia, J. (1999). Adaptação e análise factorial confirmatória da Rosenberg self-esteem scale com uma amostra e adolescentes: Resultados preliminares. Actas da VI Conferência Internacional de Avaliação Psicológica: Formas e Contextos (pp. 101-103). Braga: Apport.

Santos, P. J., \& Maia, J. (2003). Análise factorial confirmatória e validação preliminar de uma versão portuguesa da escala de autoestima de Rosenberg. Psicologia: Teoria, Investigação e Prática, 2, 253-268.
Schieman, S., \& Campbell, J.E. (2001). Age Variations in Personal Agency and Self-Esteem: The Context of Physical Disability. Journal of Aging and Health, 13, 155-185.

Schmitt, D. P., \& Allik, J. (2005). Simultaneous administration of the Rosenberg self-esteem scale in 53 nations: Exploring the universal and culture-specific features of global self-esteem. Journal of Personality and Social Psychology, 89, 623-642.

Sonstroem, R. J., \& Morgan, W.P. (1989). Exercise and self-esteem: Rationale and model. Medicine and Science in Sports and Exercise, 21, 329-337.

Spence, J. C., Mcgannon, K. R., \& Poon, P. (2005). The Effect of Exercise on Global Self-Esteem: A Quantitative Review. Journal of Sport \& Exercise Psychology, 27, 311-334.

Streiner, D. L., \& Norman, G. R. (2007). Health Measurement Scales: A practical guide to their development and use. New York: Oxford University Press.

Tafarodi, R. W., \& Milne, A. B. (2002). Decomposing global self-esteem. Journal of Personality, 70, 443-483.

U.S. Department of Health and Human Services, Centers for Disease Control and Prevention, National Center for Chronic Disease Prevention and Health Promotion. (1996). Physical activity and health: A Report of the Surgeon General. Retrieved November 12, 2008 from: www.cdc. gov/nccdphp/sgr/pdf/chap5.pdf

Wilson, P. M., \& Rodgers, W. M. (2002). The relationships between exercise motives and physical self-esteem in female exercise participants: An application of self-determination theory. Journal of Applied Biobehavioral Research, 7, 30-43.

(cc)EY-No Todo o conteúdo da revista Motricidade está licenciado sob a Creative Commons, exceto quando especificado em contrário e nos conteúdos retirados de outras fontes bibliográficas. 